\title{
Primer informe del género Gambierdiscus (Dinophyceae) y otros dinoflagelados bentónicos en el Parque Nacional Isla del Coco, Costa Rica, Pacífico Tropical Oriental
}

\author{
Maribelle Vargas-Montero ${ }^{1,2}$, Alvaro Morales ${ }^{3,4}$ \& Jorge Cortés ${ }^{3,4}$ \\ 1. Centro de Investigación en Estructuras Microscópicas (CIEMic), Ciudad de la Investigación, Universidad de Costa \\ Rica, San Pedro, 11501-2060 San José, Costa Rica; maribelle.vargas@ucr.ac.cr \\ 2. Facultad de Microbiología, Universidad de Costa Rica, San Pedro, 11501-2060 San José, Costa Rica; \\ 3. Centro de Investigación en Ciencias del Mar y Limnología (CIMAR), Ciudad de la Investigación, Universidad de \\ Costa Rica, San Pedro, 11501-2060 San José, Costa Rica; alvaro.morales@ucr.ac.cr, jorge.cortes@ucr.ac.cr \\ 4. Escuela de Biología, Universidad de Costa Rica, San Pedro, 11501-2060 San José, Costa Rica
}

$$
\text { Recibido 02-III-2012. Corregido 20-VII-2012. Aceptado 24-IX-2012. }
$$

\begin{abstract}
First report of the genus Gambierdiscus (Dinophyceae) and other benthic dinoflagellates from Isla del Coco National Park, Costa Rica, Eastern Tropical Pacific. The Eastern Tropical Pacific is a region reported as free of ciguatera poisoning that causes serious gastrointestinal, neural and cardiovascular problems, even death. But with this study we found a high incidence of benthic microalgae involved in ciguatera poisoning in Isla del Coco National Park (PNIC, from its name in Spanish), Costa Rica. Between 2006 and 2011, during expeditions to PNIC, 420 phytoplankton samples with the interest of finding benthic dinoflagellates involved in the ciguatera poisoning were collected and analyzed. Samples were taken with phytoplankton nets, towed vertically and horizontally or carried by diving, between 5 to $30 \mathrm{~m}$ depth, over reef areas, and by direct extraction from benthic macroalgae. We found the dinoflagellates Gambierdiscus spp., Coolia tropicalis, Coolia $\mathrm{cf}$. areolota, Prorocentrum concavum, Prorocentrum compressum, Amphidinium carterae and Ostreopsis siamensis. The quantity of dinoflagellates by macroalgae weight was high, mainly for Gambierdiscus. Ostreopsis and Prorocentrum, the most widely distributed genera throughout the collection sites. Gambierdiscus is a ciguatera producing genus. Two different sizes of Gambierdiscus were found, and comparing our samples with other studies, we conclude that they are different to any previously reported. They possibly represent two new species. Coco Island is an oceanic island and because of its protection status, it is an ideal site for studying the evolution of marine phytoplankton. Also, long-term monitoring is important due to the variety of potentially toxic dinoflagellates living in this marine ecosystem. This is the first study to report benthic dinoflagellates implicated in ciguatera poisoning in other areas of the Eastern Tropical Pacific. Citation: Vargas-Montero, M., Á. MoralesRamírez \& J. Cortés. 2012. Primer informe del género Gambierdiscus (Dinophyceae) en el Parque Nacional Isla del Coco, Pacífico Tropical Oriental. Rev. Biol. Trop. 60 (Suppl. 3): 187-199. Epub 2012 Dec 01.
\end{abstract}

Key words: Ciguatera, Gambierdiscus, benthic dinoflagellates, Pacific Ocean, Isla del Coco, Costa Rica.

El Parque Nacional Isla del Coco, núcleo del Área de Conservación Marina Isla del Coco, se encuentra ubicado a $532 \mathrm{~km}$ al suroeste del litoral Pacífico de Costa Rica $\left(5^{\circ} \mathrm{N}-87^{\circ} \mathrm{W}\right)$. Tiene un área terrestre de $24 \mathrm{~km}^{2}$ y $1997 \mathrm{~km}^{2}$ de área de ecosistemas marinos protegidos. Está isla oceánica tiene una edad de aproximadamente 2 millones de años y es el único punto que emerge a la superficie del océano de la Cordillera Submarina de Coco, desarrollada en dirección suroeste-noreste sobre la Placa de Coco (Cortés 2008, Rojas \& Alvarado 2012).

La Isla del Coco está bajo la influencia de un complejo sistema de corrientes marinas, entre ellas, la Contracorriente Ecuatorial del Norte proveniente del oeste (Indo-Pacífico), la cual transporta aguas tropicales cálidas ligeramente al norte de la línea ecuatorial. Además, 
en la Isla del Coco se manifiesta, periódicamente, el fenómeno de interacción oceánica y atmosférica conocido como El Niño (Fiedler \& Talley 2006, Lizano 2008). El Niño ejerce una fuerte influencia en la composición y distribución de especies del fitoplancton marino, principalmente en la zona ecuatorial, afectando la presencia de especies que se encuentran siempre en la columna de agua. Este fenómeno es un evento dominante en la variabilidad del clima interanual en el Pacífico Tropical y que tiene efectos profundos en las condiciones oceanográficas, atmosféricas y también en el ambiente biológico, incluyendo las comunidades fitoplanctónicas (Morales \& Brugnoli 2001, Pennington et al. 2006) y en algunas ocasiones favoreciendo la presencia de especies nocivas de fitoplancton (Franks \& Anderson 1992, Sar et al. 2002, Hallegraeff 2003).

Todas estas corrientes provenientes de diferentes zonas geográficas transportan especies de fitoplancton hacia la isla, de tal forma que se produce el trasiego natural de microorganismos que podrían encontrar en la isla un ambiente apto para su desarrollo y a su vez estas especies podrían ser nocivas para el ecosistema (Fukuyo 1982, Glibert \& Pitcher 2001).

A la fecha se desconoce la distribución de dinoflagelados potencialmente tóxicos en el área del Parque Nacional Isla del Coco. Esta es una situación de interés científico, ya que varias especies de microalgas desarrollan extensas proliferaciones que pueden ser nocivas, debido a la producción en algunos casos, de toxinas que producen envenenamientos en la vida humana y marina de manera directa o indirecta (Hallegraeff 1993).

Especies de Gambierdiscus son conocidas por producir ciaguatoxinas (P-CTX o C-CTX, Lewis \& Holmes 1993), que a su vez originan la enfermedad conocida como ciguatera, ocasionada por el consumo de peces contaminados con ciguatoxinas. El hábito béntico/epifítico principalmente del género Gambierdiscus, (Anderson \& Lobel 1987), puede provocar acumulación de ciaguatoxinas en peces herbívoros que consumen macroalgas conteniendo dinoflagelados tóxicos (Steidinger 1993) y este a su vez es consumido por un pez carnívoro como la barracuda. Las ciguatoxinas se acumulan en sus músculos y se pone en riesgo a los consumidores humanos (Lewis \& Holmes 1993). Existen más de 175 síntomas gastrointestinales, neurotóxicos o cardiovasculares asociados con esta toxina (Becker \& Sanders 1991). Esta intoxicación por consumo de organismos contaminados puede producir problemas gastrointestinales y enfermedades neurológicas y en casos extremos la persona puede morir por problemas respiratorios, sin embargo, a pesar de la alta incidencia, la mortalidad humana es baja (Hallegraeff 1993). Asimismo, se conoce que otras especies de dinoflagelados bentónicos están implicadas con la producción de toxinas (Tindall \& Morton 1998), como son algunas especies de Coolia (Meunier, 1917), Ostreopsis (Schmidt, 1901) y Prorocentrum (Ehrenberg, 1833).

Este trabajo describe por primera vez algunas de las especies de dinoflagelados bentónicos potencialmente tóxicos presentes en las aguas alrededor del Parque Nacional Isla del Coco.

\section{MATERIALES Y MÉTODOS}

Diez campañas oceanográficas al Parque Nacional Isla del Coco, entre el 2006 y 2011, permitieron la colecta de 420 muestras de fitoplancton marino en diversas estaciones ubicadas alrededor de la isla (Cuadro 1). Para el objetivo de este manuscrito, se analizaron 36 muestras, algunas tomadas con red de fitoplancton de $20 \mu \mathrm{m}$ de apertura de malla y otras mediante buceos. Las muestras se tomaron entre los 5 y los $30 \mathrm{~m}$ de profundidad en zonas de arrecife y por extracción directa de macroalgas (Cuadro 1).

Todas las muestras se fijaron con una mezcla de lugol ácido, se sedimentaron en el laboratorio y fueron observadas entre los 400 y 1000 aumentos utilizando un microscopio invertido marca Olympus modelo IX-51 equipado con una cámara digital DP52. Las muestras donde se observó la presencia de dinoflagelados bentónicos se limpiaron mediante diversos lavados 
CUADRO 1

Datos de las muestras analizadas

TABLE 1

Information of the analyzed samples

\begin{tabular}{|c|c|c|c|c|}
\hline $\begin{array}{c}\text { Número de } \\
\text { muestra }\end{array}$ & $\begin{array}{c}\text { Fecha de } \\
\text { colecta }\end{array}$ & Localidad & $\begin{array}{l}\text { Profundidad de } \\
\text { colecta }(\mathrm{m})\end{array}$ & Coordenadas \\
\hline IC-01, IC-02 & Set-2006 & Cabo Atrevido & 5 & $05^{\circ} 31^{\prime} 99^{\prime \prime} \mathrm{N}-87^{\circ} 01^{\prime} 55^{\prime \prime} \mathrm{W}$ \\
\hline IC-03, IC-04 & Set-2006 & Bahía Chatham & 5 & $05^{\circ} 32^{\prime} 29^{\prime \prime} \mathrm{N}-87^{\circ} 02^{\prime} 43^{\prime \prime} \mathrm{W}$ \\
\hline IC-05, IC-06 & Set-2006 & Dos Amigos & 5 & $05^{\circ} 30^{\prime} 51^{\prime \prime} \mathrm{N}-87^{\circ} 05^{\prime} 71^{\prime \prime} \mathrm{W}$ \\
\hline IC-27, IC-28 & Oct-2007 & Vikinga & 20 & \\
\hline IC-31, IC-32 & Oct-2007 & Manuelita & 30 & $5^{\circ} 33^{\prime} 25.1^{\prime \prime} \mathrm{N}-87^{\circ} 03^{\prime} 03.7^{\prime \prime} \mathrm{W}$ \\
\hline IC-38 & Oct-2007 & Cabo Atrevido & 20 & $05^{\circ} 31^{\prime} 99^{\prime \prime} \mathrm{N}-87^{\circ} 01^{\prime} 55^{\prime \prime} \mathrm{W}$ \\
\hline IC-41 & Oct-2007 & Punta Iglesias & 20 & $05^{\circ} 30^{\prime} 64^{\prime \prime} \mathrm{N}-87^{\circ} 03^{\prime} 92^{\prime \prime} \mathrm{W}$ \\
\hline IC-43 & Oct-2007 & Dos Amigos & 20 & $05^{\circ} 30^{\prime} 51^{\prime \prime} \mathrm{N}-87^{\circ} 05^{\prime} 70^{\prime \prime} \mathrm{W}$ \\
\hline IC-45 & Oct-2007 & Punta Maria & 20 & $05^{\circ} 32^{\prime} 10^{\prime \prime} \mathrm{N}-87^{\circ} 05^{\prime} 30^{\prime \prime} \mathrm{W}$ \\
\hline IC-48 & Oct-2007 & Bahía Wafer & 12 & $05^{\circ} 32^{\prime} 80^{\prime \prime} \mathrm{N}-87^{\circ} 03^{\prime} 75^{\prime \prime} \mathrm{W}$ \\
\hline *IC-64 & Oct-2007 & Punta Ulloa & 6 & $5^{\circ} 33^{\prime} 05.5 \mathrm{~N}-87^{\circ} 02^{\prime} 09.0^{\prime \prime} \mathrm{W}$ \\
\hline *IC-65 & Oct-2007 & Punta Ulloa & 15 & $5^{\circ} 33^{\prime} 05.5 \mathrm{~N}-87^{\circ} 02^{\prime} 09.0^{\prime \prime} \mathrm{W}$ \\
\hline IC-84 & Oct-2007 & Manuelita & 5 & $5^{\circ} 33^{\prime} 25.1^{\prime \prime} \mathrm{N}-87^{\circ} 03^{\prime} 03.7^{\prime \prime} \mathrm{W}$ \\
\hline IC-149 & Mar-2009 & Bahía Chatham & 15 & $05^{\circ} 32^{\prime} 29^{\prime \prime} \mathrm{N}-87^{\circ} 02^{\prime} 43^{\prime \prime} \mathrm{W}$ \\
\hline IC-150 & Mar-2009 & Bahía Chatham & 12 & $05^{\circ} 32^{\prime} 29^{\prime \prime} \mathrm{N}-87^{\circ} 02^{\prime} 43^{\prime \prime} \mathrm{W}$ \\
\hline IC-151 & Mar-2009 & Bahía Chatham & 9 & $05^{\circ} 32^{\prime} 29^{\prime \prime} \mathrm{N}-87^{\circ} 02^{\prime} 43^{\prime \prime} \mathrm{W}$ \\
\hline IC-152 & Mar-2009 & Bahía Chatham & 6 & $05^{\circ} 32^{\prime} 29^{\prime \prime} \mathrm{N}-87^{\circ} 02^{\prime} 43^{\prime \prime} \mathrm{W}$ \\
\hline IC-153 & Mar-2009 & Bahía Chatham & 3 & $05^{\circ} 32^{\prime} 29^{\prime \prime} \mathrm{N}-87^{\circ} 02^{\prime} 43^{\prime \prime} \mathrm{W}$ \\
\hline$* I C-205$ & Mar-2009 & Punta Ulloa & 14 & $5^{\circ} 33^{\prime} 05.5 \mathrm{~N}-87^{\circ} 02^{\prime} 09.0^{\prime \prime} \mathrm{W}$ \\
\hline IC-235 & Abr-2010 & Bahía Wafer & 15 & $05^{\circ} 33^{\prime \prime} 23 \mathrm{~N}-87^{\circ} 02^{\prime} 39^{\prime \prime} \mathrm{W}$ \\
\hline IC-238 & Abr-2010 & Manuelita & 15 & $5^{\circ} 33^{\prime} 25.1^{\prime \prime} \mathrm{N}-87^{\circ} 03^{\prime} 03.7^{\prime \prime} \mathrm{W}$ \\
\hline IC-391 & Jul-2011 & Bahía Wafer & 25 & $05^{\circ} 32^{\prime} 80^{\prime \prime} \mathrm{N}-87^{\circ} 03^{\prime} 75^{\prime \prime} \mathrm{W}$ \\
\hline *IC-394 & Jul-2011 & Punta Ulloa & 30 & $5^{\circ} 33^{\prime} 05.5 \mathrm{~N}-87^{\circ} 02^{\prime} 09.0^{\prime \prime} \mathrm{W}$ \\
\hline IC-395 & Jul-2011 & Punta Yglesias & 30 & $05^{\circ} 30^{\prime} 64^{\prime \prime} \mathrm{N}-87^{\circ} 03^{\prime} 92^{\prime \prime} \mathrm{W}$ \\
\hline IC-396 & Jul-2011 & Punta Gissler & 30 & $05^{\circ} 33^{\prime} 0^{\prime \prime} \mathrm{N}-87^{\circ} 04^{\prime} 02^{\prime \prime} \mathrm{W}$ \\
\hline IC-409 & Jul-2011 & Dos Amigos & 30 & $05^{\circ} 30^{\prime} 51^{\prime \prime} \mathrm{N}-87^{\circ} 05^{\prime} 71^{\prime \prime} \mathrm{W}$ \\
\hline IC-411 & Jul-2011 & Manuelita & 10 & 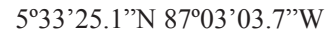 \\
\hline IC-412 & Jul-2011 & Bahía Weston & 20 & $05^{\circ} 33^{\prime} 15^{\prime \prime} \mathrm{N}-87^{\circ} 03^{\prime} 32^{\prime \prime} \mathrm{W}$ \\
\hline IC-417 & Jul-2011 & Punta María & 30 & $05^{\circ} 32^{\prime} 10^{\prime \prime} \mathrm{N}-87^{\circ} 05^{\prime} 30^{\prime \prime} \mathrm{W}$ \\
\hline IC-419 & Jul-2011 & Bajo Alcyone & 30 & $05^{\circ} 30^{\prime} 80^{\prime \prime} \mathrm{N}-87^{\circ} 01^{\prime} 85^{\prime \prime} \mathrm{W}$ \\
\hline IC-420 & Jul-2011 & Cabo Atrevido & 30 & $05^{\circ} 31^{\prime} 99^{\prime \prime} \mathrm{N}-87^{\circ} 01^{\prime} 55^{\prime \prime} \mathrm{W}$ \\
\hline
\end{tabular}

* Muestras tomadas mediante buceos, el resto mediante redes para fitoplancton.

con detergente no iónico Triton $\mathrm{X}-100 \AA$ y agua destilada. Posteriormente se secaron al aire y se cubrieron con una capa de $40 \mu \mathrm{m}$ de platino-paladio para aumentar su conductividad y poder ser observados en los microscopios electrónicos de barrido marca Hitachi modelos S-570 y S-2360N, donde se tomaron fotografías entre los 1000 y 5000 aumentos.

\section{RESULTADOS}

En 14 de las muestras se encontraron varias especies de dinoflagelados bentónicos, dos especies de Gambierdiscus (Figs. 1, 2), Coolia tropicalis Faust, 1995 (Fig. 3, 4), Coolia cf. areolota (Fig. 4), Prorocentrum compressum (Bailey, 1850) Abé ex Dodge, 1975 

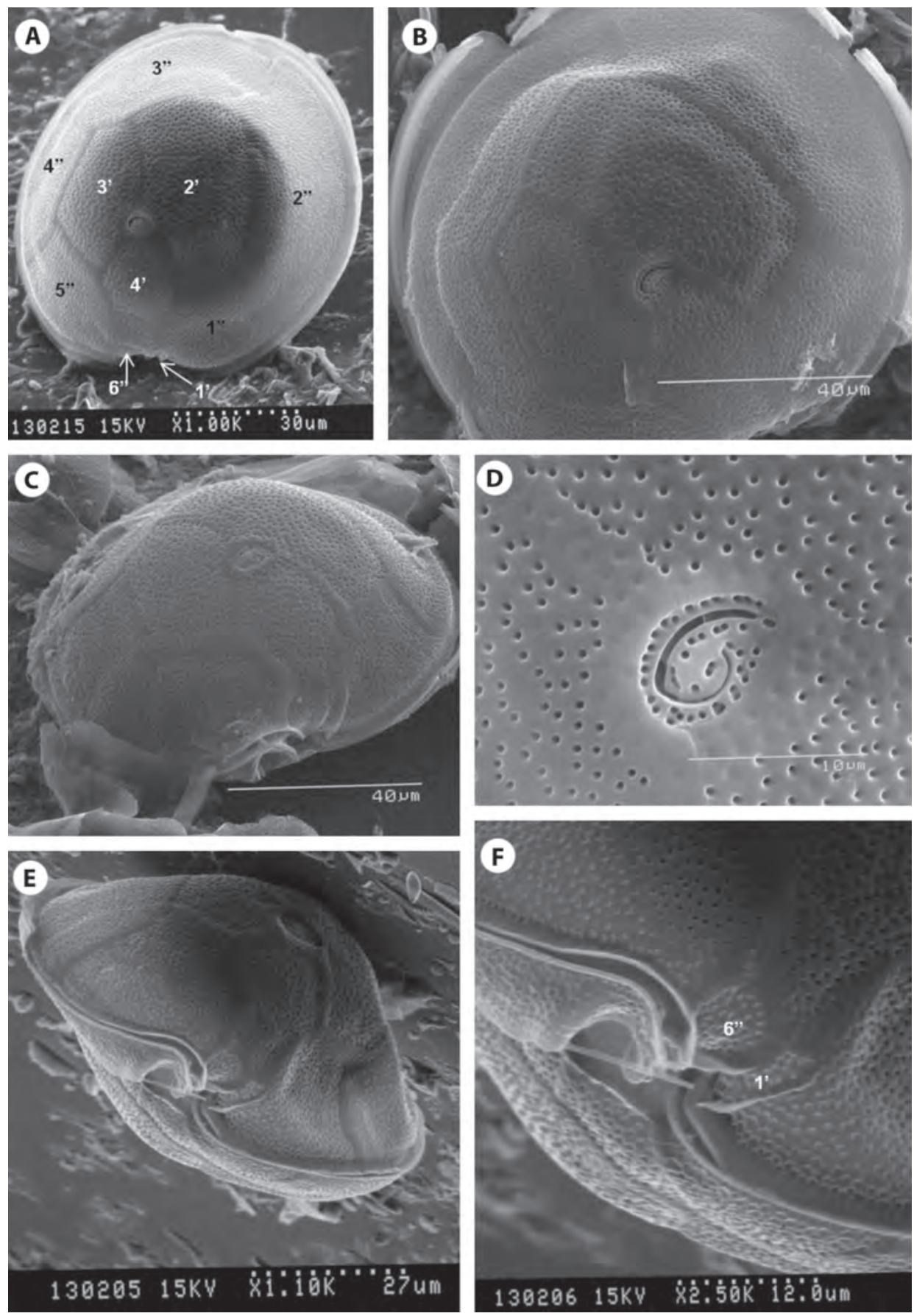

Fig. 1. (a-c) Gambierdiscus spp., vista apical, (d) detalle. (e-f) Vista lateral, complejo del poro apical y posición de las placas 1'y 6".

Fig. 1. (a-c) Gambierdiscus spp. apical view, (d) detail. (e-f) Lateral view, apical pore complex, and plates 1' and 6". 

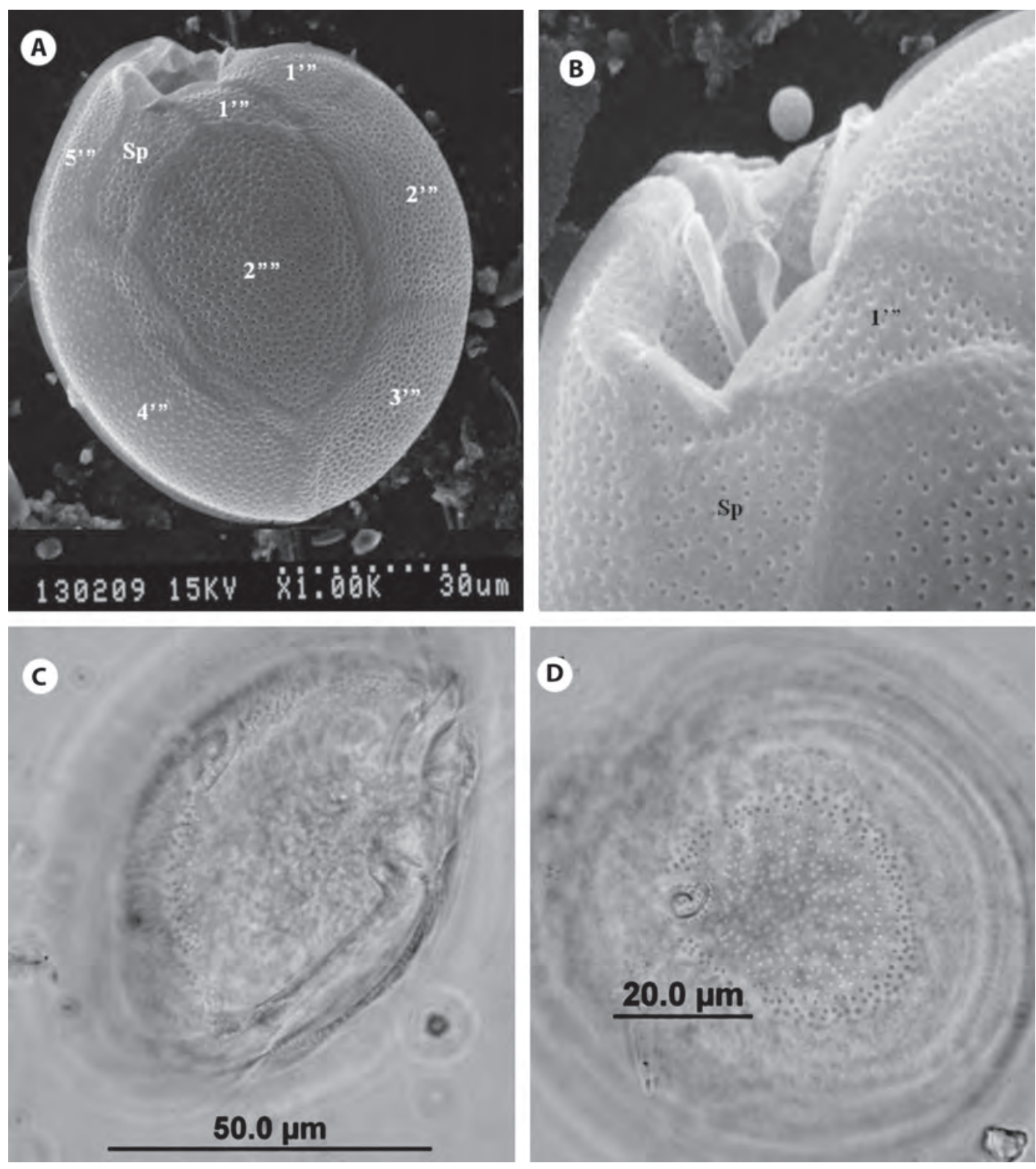

Fig. 2. (a-b) Gambierdiscus spp. vista de la hipoteca, con indicación de la nomenclatura de las placas. (c) Vista ventral. (d) Vista de la hipoteca con la posición del Po.

Fig. 2. (a-b) Gambierdiscus sp. hypothecal view. (c) Ventral view. (d) Antapical view with Po.

(Fig. 5), Prorocentrum concavum Fukuyo, 1981 (Fig. 5), Ostreopsis siamensis Schmidt, 1901 (Fig. 6), Amphidinium carterae Hulburt, 1957 (Fig. 6). Las muestras donde se encontraron las especies del género Gambierdiscus se colectaron en octubre 2007 y en abril 2008 en buceos entre los 6 y $15 \mathrm{~m}$ de profundidad en Punta Ulloa, Bahía Chatham y Bahía Weston. Durante la colecta del 2007 se extrajo la macroalga Sargassum filipendula C. Agardh, 

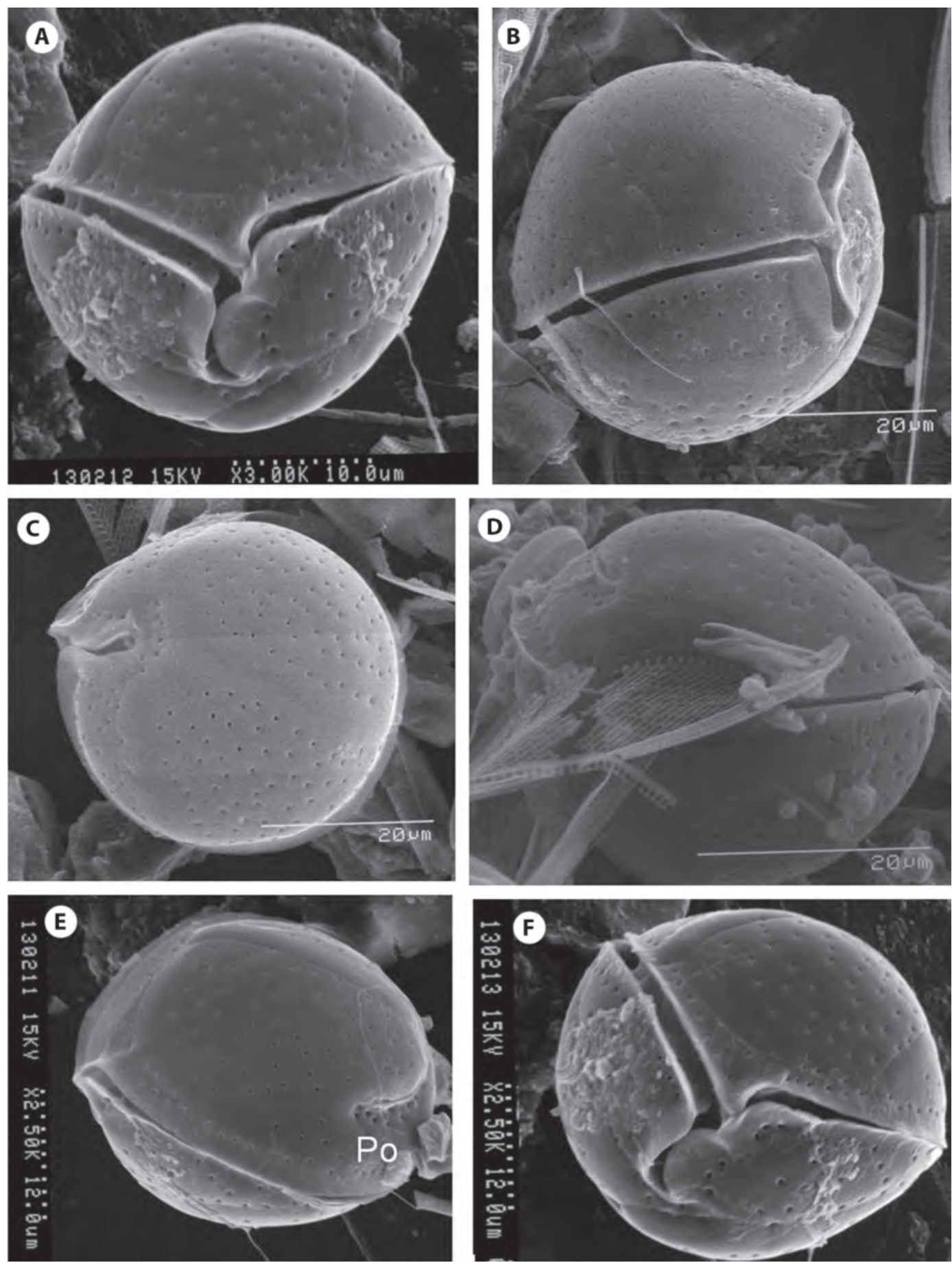

Fig. 3. Coolia tropicalis. (a, b, f) Vista ventral. (c)Vista de la hipoteca. (d) Vista lateral. (e) Vista dorsal con el Po. Fig. 3. Coolia tropicalis. (a, b, f) Ventral view. (c) Hypothecal view. (d) Lateral view. (e) Dorsal view with Po. 

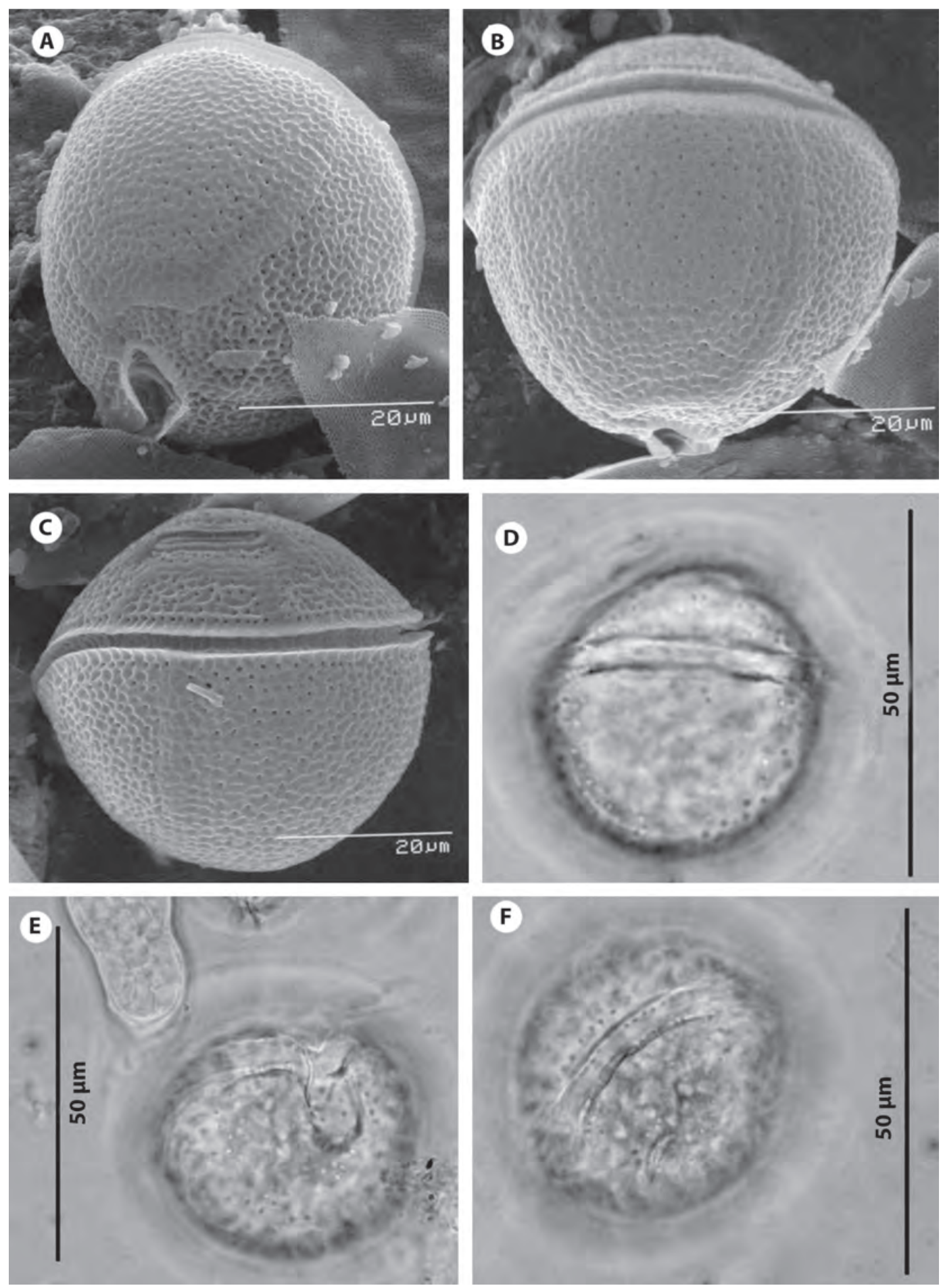

Fig. 4. (a-c) Coolia cf. areolata. (d-f) Coolia tropicalis. Fig. 4. (a-c) Coolia cf. areolata. (d-f) Coolia tropicalis. 

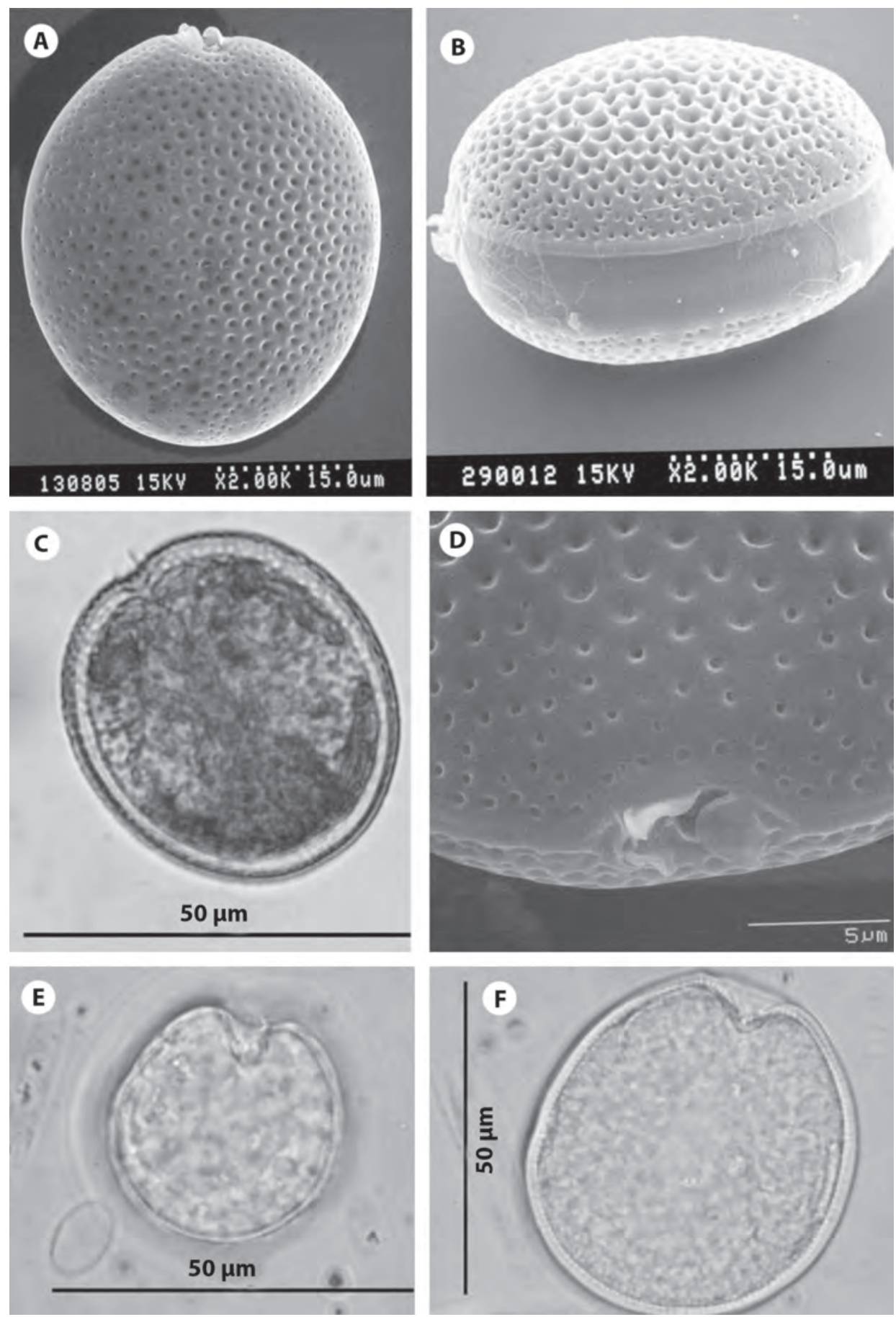

Fig. 5. (a, b, c, d) Prorocentrum compressum. (e-f) Prorocentrum concavum.

Fig. 5. (a, b, c, d) Prorocentrum compressum. (e-f) Prorocentrum concavum. 

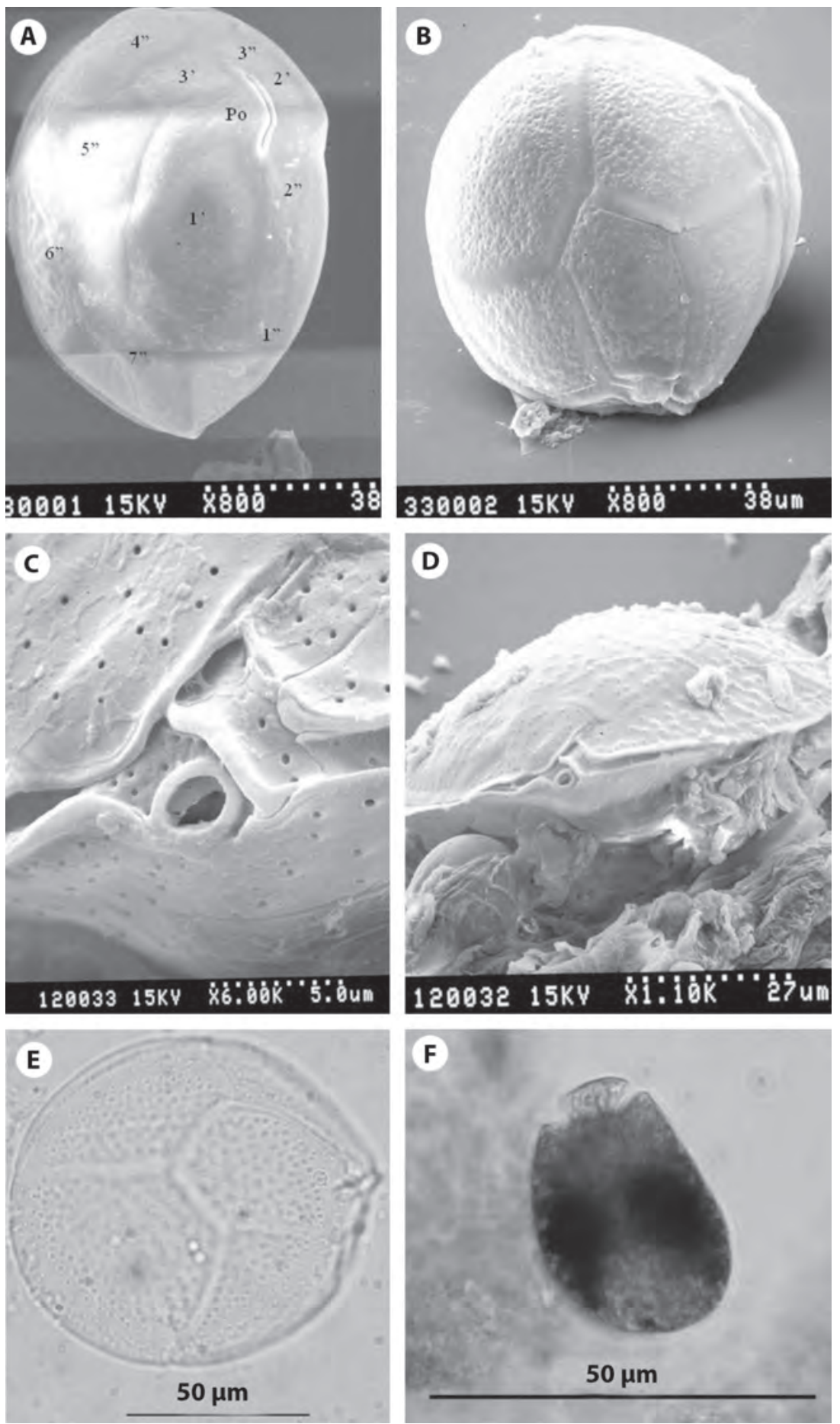

Fig. 6. (a-d) Ostreopsis siamensis. (a) Vista de la epiteca donde se observa el poro apical Po. (b) Vista de la hipoteca. (c-d) Localización de la apertura ventral. (e) Amphidinium carterae. (f) Vista de la hipoteca de O. siamensis.

Fig. 6. (a-d) Ostreopsis siamensis. (a) Epithecal view with the apical pore plate Po. (b) Hypothecal view. (c-d) Ventral view showing the location of ventral opening. (e) Amphidinium carterae. (f) Hypothecal view of $O$. siamensis. 
CUADRO 2

Distribución de los dinoflagelados bentónicos alrededor de la Isla del Coco

TABLE 2

Distribution of benthic dinoflagellates around Coco Island

\begin{tabular}{|c|c|c|c|c|c|c|}
\hline Estaciones/Especie & $\begin{array}{c}\text { Amphidinium } \\
\text { carterae }\end{array}$ & $\begin{array}{c}\text { Coolia } \\
\text { tropicalis }\end{array}$ & $\begin{array}{c}\text { Gambierdiscus } \\
\text { spp. }\end{array}$ & $\begin{array}{c}\text { Ostreopsis } \\
\text { siamensis }\end{array}$ & $\begin{array}{c}\text { Prorocentrum } \\
\text { concavum }\end{array}$ & $\begin{array}{c}\text { Prorocentrum } \\
\text { compressum }\end{array}$ \\
\hline Punta Ulloa & & IC-64,65 & IC-64, 65 & & IC-64, 65 & \\
\hline Bahía Chatham & IC-238 & & IC-238 & IC-03,238 & IC-238 & $\mathrm{I}-238,03$ \\
\hline Manuelita & IC-31, 32 & & & & IC-31, 32 & IC-31, 32 \\
\hline Vikinga & & & & IC-27,28 & & \\
\hline Bahía Wafer & IC-235 & & IC-235 & IC-235 & IC-235,391 & IC-235 \\
\hline Bahía Weston & & & IC-412 & IC-412 & IC-412 & \\
\hline Bajo Alcyone & & & & IC-419 & & \\
\hline Punta María & & & & & IC-45 & \\
\hline Punta Iglesias & & & & & IC-41 & \\
\hline
\end{tabular}

1824 y por agitación de la macroalga se extrajeron los dinoflagelados epífitos. Se realizó un conteo de dinoflagelados por peso de macroalga, y se determinó que en un gramo de macroalga húmeda se encontraba un promedio de 15 dinoflagelados del género Gambierdiscus, 8 del género Coolia y 4 del género Prorocentrum, así como altas concentraciones de diatomeas. Los géneros Ostreopsis y Prorocentrum mostraron tener la mayor distribución en los sitios de colecta, ya que se encontraron en diversas estaciones de la isla, Bahía Chatham, Manuelita, Bahía Wafer, Bahía Weston, Bajo Alcyone, Punta María y Punta Iglesias (Cuadro 2).

\section{Morfología del género Gambierdiscus}

Para identificar al género Gambierdiscus en este estudio se utilizo el sistema de tabulación de las placas modificado de Kofoid (1909) utilizado por Fraga et. al. (2011) lo cual se refiere al conteo de las placas en series designadas específicamente. Las mediciones de los organismos así como la tabulación de sus placas se realizó con las fotografías tomadas en el microscopio electrónico. Se tomaron promedios en las mediciones de los ejes dorsoventral y del eje antero-posterior de la célula para medir su tamaño además, se enumeraron sus placas considerando en la epiteca las placas apicales con la simbología ', la placa donde se localiza el poro apical como Po, las placas precingulares con la simbología "; en la hipoteca las placas postcingulares como ", antiapicales con "” y la sulcal posterior conocida como S.p.

Reino Chromista

Subreino Harosa

Phylum Myzozoa

Subphylum Dinozoa

Clase Peridinea

Subclase Gonyaulacoidia

Orden Gonyaulacida

Familia Goniodomataceae

Género Gambierdiscus Adachi \& Fukuyo, 1979

Dinoflagelado tecado, fotosintético, con muchos cloroplastos alargados alrededor del núcleo, el cuál es redondeado, posicionado en la parte dorsal y está desplazado ventralmente hacia la derecha de la célula. Las células de Gambierdiscus son de forma lenticular, anteroposteriormente comprimidas como la mayoría de los miembros del género. Tienen un tamaño promedio en su eje dorso-ventral de $81.5 \mu \mathrm{m}$ de ancho y $81 \mu \mathrm{m}$ de largo, midiendo $104 \mu \mathrm{m}$ de ancho y $92 \mu \mathrm{m}$ de largo las células más grandes encontradas. Otros organismos fueron encontrados en la misma muestra, solo que mucho más pequeños con un tamaño promedio de $63.7 \mu \mathrm{m}$ de ancho y $60.8 \mu \mathrm{m}$ de largo y serán estudiados en el futuro. 
La célula es oval vista tanto apical como anti apicalmente, con un hundimiento en el área flagelar rodeado por un lóbulo al lado derecho de la célula. La epiteca y la hipoteca son similares en altura, las placas tecales son finas cubiertas con poros evidentes de la misma forma y un tamaño de $5 \mu \mathrm{m}$ de diámetro en promedio, distribuidos de forma irregular y sin otra ornamentación. La fórmula tecal es Po, 4', 0a, 6", 5"', 0p, 2"', sin posibilidad de analizar las placas sulcales conocidas con la simbología s. ni las placas cingulares. La placa del poro apical Po es oval, rodeada por poros y tiene forma de anzuelo como el resto de los miembros del género Gambierdiscus. . La primera placa apical 1' es alargada y es la placa más pequeña de la epiteca y está rodeada de poros orientados en filas; no toca la placa Po pero si toca la 4' igual que la placa 6" y ambas poseen pequeñas alas. Las placas 1' y 6" son las más pequeñas y están inclinadas hacia la región ventral de la célula justo en la torsión del área flagelar. La placa 4' está compuesta por siete vértices. La placa 2' es más o menos rectangular, la placa 2 " es la más grande de la serie precingular y junto con 3" son elongadas y ocupan la mayor parte dorsal de la serie. Las placas 3' y 4' son de un tamaño similar y más pequeñas que 2 '.

El cíngulo es descendente un ancho en diámetro y la hipoteca está compuesta por dos placas anti apicales y 5 poscingulares junto con la placa S.p. quien está al lado del sulcus y considerada como la placa 2 "' por algunos autores, con cinco lados y conectada en forma triangular con las placas 3"' y 4"'.

En la hipoteca la placa S.p. se encuentra fuera del área sulcal y la placa 2"' es la más grande y ocupa la mayor parte de la hipoteca. La placa poscingular más grande es la 4" la cual casi ocupa en su totalidad el lado derecho de la hipoteca, siendo la placa 5" de forma arqueada y la más pequeña de la serie.

Las muestras preservadas (IC-64 e IC-65) se encuentran en la colección de fitoplancton marino en el CIEMic de la Universidad de Costa Rica y fueron colectadas de la macroalga S. filipendula.

\section{DISCUSIÓN}

La distribución global de ciguatera abarca áreas en el Caribe, Australia y la Polinesia Francesa, pero ninguno de ellos incluye la presencia de esta intoxicación o de los dinoflagelados productores en el Pacífico Tropical Oriental y mucho menos en Costa Rica (Hallegraeff et al. 2003, Litaker et al. 2010, Tester et al. 2010, Rhodes 2011). En esta investigación encontramos en la Isla del Coco una alta incidencia de microalgas bentónicas implicadas en la intoxicación por ciguatera a nivel mundial. Es conocido que muchos dinoflagelados de origen bentónico son productores de toxinas, como los dinoflagelados del género Coolia (Yasumoto et al. 1987) y muchas especies del género Gambierdiscus (G. australes, G. pacificus, G. polynisensis, G. toxicus (Yasumoto et al. 1979, Chinain et al. 1999) y $G$. excentricus (Fraga et al. 2011). A pesar de que no se han realizado estudios de toxicidad a las especies de dinoflagelados bentónicos presentes en el Parque Nacional Isla del Coco, es probable que algunas de estas especies reportadas en este estudio, sean microalgas productoras de toxinas,

Esta es la primera vez que el género Gambierdiscus, bien conocido por contener la mayor cantidad de especies productoras de ciguatoxinas, se informa para el Pacífico Tropical Oriental, y se describe su morfología. Se ha comparado su taxonomía con la de G. toxicus, G. caribaeus, G. carolinianus, G. carpenteri, G. ruetzleri, G. yasumotoi, G. australes, G. belizeanus, $G$. pacificus y $G$. polynesiensis, descritas en forma detallada por Litaker et al. (2009) y con G. excentricus descrita por Fraga et al. (2011). Después del análisis taxonómico realizado, se podría pensar que la especie de Gambierdiscus encontrada en el Parque Nacional Isla del Coco, no corresponde a ninguna de las especies descritas anteriormente y que podría tratarse de una nueva especie, sin embargo, esto se podrá confirmar posteriormente a través de cultivos y análisis moleculares.

Analizando la nomenclatura para las placas tecales que realizan ambos autores, Litaker 
et al. (2009) y Fraga et al. (2011), tomando en cuenta la discrepancia que existe entre diferentes autores sobre la nomenclatura para describir la tabulación del género Gambierdiscus, se aceptó la interpretación del Sistema de Nomenclatura Kofoideana sugerida en Fraga et al. (2011) donde se considera en la epiteca como primera placa apical (1') a la placa considerada por muchos autores como primera placa precingular (1") y en la hipoteca la segunda placa antiapical 2"" conocida como 1p y la sulcal posterior (S.p.) en lugar de la segunda placa antiapical 2"', tomando que se acepta la placa 1 ' a pesar de que esta no toca a Po como se acepta también en el caso de los dinoflagelados del género Alexandrium.

La especie de Gambierdiscus de este estudio se diferencia de otras especies por su forma, tamaño, relación de altura entre las placas de la epiteca 2'/3' y 2'/4', otros detalles como la forma de las placas, la posición que ocupa el poro apical Po y la forma de la placa 2', por lo que será estudiada en el futuro.

Al constituir la Isla del Coco uno de los sitios naturales privilegiados a nivel mundial debido a su riqueza biológica, aislamiento y estado de conservación, la hacen un sitio ideal para el estudio de evolución de especies de fitoplancton marino y su monitoreo a largo plazo, y queda de manifiesto la importancia de este estudio por la variedad de dinoflagelados bentónicos potencialmente tóxicos que habitan este ecosistema marino.

\section{AGRADECIMIENTOS}

Este estudio fue posible gracias al apoyo financiero del "Fonds Français pour l'Environnement Mondial (FFEM)", del Consejo Nacional de Rectores de las Universidades Públicas de Costa Rica (CONARE), del apoyo logístico del Área de Conservación Marina Isla del Coco (ACMIC), del Undersea Hunter Group y de la Vicerrectoría de Investigación de la Universidad de Costa Rica por su apoyo a los proyectos 808-B0-060, 808-B0-654 y 810-B1150.Un especial agradecimiento a Eleazar Ruiz por su apoyo en el campo.

\section{RESUMEN}

El Pacífico Tropical Oriental se ha indicado como una región de los océanos libre de ciguatera, una intoxicación que causa serios trastornos gastrointestinales, neurológicos y cardiovasculares, en casos extremos, la muerte. Pero con el presente estudio hemos encontrado una incidencia alta de microalgas bénticas involucradas en la producción de ciguatera en el Parque Nacional Isla del Coco (PNIC), Costa Rica. En campañas oceanográficas realizadas entre el 2006 y el 2011 al PNIC se recolectaron 420 muestras de fitoplancton con el interés de encontrar especies de dinoflagelados bentónicos implicados en la producción de ciguatera. Las muestras se tomaron con red de fitoplancton o mediante buceos, entre los 5 y $30 \mathrm{~m}$ de profundidad en zonas de arrecife y por extracción directa de macroalgas. Se encontró a los dinoflagelados Gambierdiscus spp., Coolia tropicales, Coolia cf. areolota, Prorocentrum concavum, Prorocentrum compressum, Amphidinium carterae y Ostreopsis siamensis. Se determinó que la cantidad de dinoflagelados por peso de macroalga era elevada, principalmente para el género Gambierdiscus. Los géneros Ostreopsis y Prorocentrum mostraron tener la mayor distribución en los sitios de colecta. Gambierdiscus es un género productor de ciguatera. Se encontraron dos tamaños diferentes de individuos de Gambierdiscus, Comparando los especímenes de Gambierdiscus encontradas con otros estudios, se concluye que son diferentes a las descritas anteriormente, por lo que podría tratarse de especies nuevas, lo que se confirmará con cultivos y análisis moleculares. Al ser la Isla del Coco un sitio oceánico aislado y en estado de conservación, es un sitio ideal para el estudio de la evolución de especies de fitoplancton marino. Además, el monitoreo a largo plazo es muy importante debido a la variedad de dinoflagelados bentónicos potencialmente tóxicos que habitan en este ecosistema marino. Este es el primer estudio donde se da a conocer la incidencia de dinoflagelados bentónicos implicados en ciguatera para el Pacífico Tropical Oriental.

Palabras clave: Ciguatera, Gambierdiscus, dinoflagelados bentónicos, Océano Pacífico, Isla del Coco, Costa Rica.

\section{REFERENCIAS}

Anderson, D.M. \& P.S. Lobel. 1987. The continuing enigma of ciguatera. Biol. Bull. 172: 89-107.

Becker, S.A. \& W.E. Sanders, Jr. 1991. Ciguatera toxins. Fla. J. Pub. Health 3: 38-41.

Chinain, M., M.A. Faust \& S. Pauillac 1999. Morphology and molecular analyses of three toxic species of Gambierdiscus (Dinophyceae): G pacificus, sp. nov., G. australes, sp. nov ., and G. polynesiensis, sp. nov. J. Phycol. 35: 1282-1296.

Cortés, J. 2008. Historia de la investigación marina de la Isla del Coco, Costa Rica. Rev. Biol. Trop. 56 (Supl. 2): $1-18$. 
Rojas, W. \& G.E. Alvarado. 2012. Geología y contexto geotectónico de la Isla del Coco y la zona marítima frente al Pacífico central de Costa Rica. Rev. Biol. Trop. 60 .

Fiedler, P.C. \& L.D. Talley. 2006 Hydrography of the eastern tropical Pacific: a review. Prog. Oceanogr. 69: $143-180$

Fraga, S., F. Rodriguez, A. Caillaud \& J. Diogène. 2011. Gambierdiscus excentricus sp. nov. (Dinophyceae), a benthic toxic dinoflagellate from the Canary Islands (NE Atlantic Ocean). Harmful Algae 11: 10-22

Franks, P.J. \& D.M. Anderson. 1992. Alongshore transport of a phytoplankton bloom in a buoyancy current: Alexandrium tamarense in the Gulf of Maine. Mar. Biol. 112: 153-164.

Fukuyo, Y. 1981. Taxonomic study on benthic dinoflagellates in coral reefs. Bull. Jap. Soc. Sci. Fish. 47: 967-978.

Fukuyo, Y. 1982. Cysts of naked dinoflagellates, pp. 205214. In T. Okaichi (Ed.). Fundamental Studies on the Effects of the Marine Environment on the Outbreaks of Red Rides. Monbusho, Tokio, Japón.

Glibert, P.M. \& G. Pitcher. 2001. Global Ecology and Oceanography of Harmful Algal Blooms. Science Plan. GEOHAB, an International Programme Sponsored by the Scientific Committee on Oceanic Research (SCOR) and the Intergovernmental Oceanographic Commission (UNESCO), París.

Hallegraeff, G.M. 1993. A review of harmful algal blooms and their apparent global increase. Phycologia 32: 79-99.

Hallegraeff, G.M., D.M. Anderson \& A.D. Cembella. 2003. Manual on Harmful Marine Microalgae: Manuals and Guides, N. 33. Intergovernmental Oceanographic Commission, UNESCO, París.

Lewis, R.J. \& M.J. Holmes. 1993. Origin and transfer of toxins involved in ciguatera. Comp. Biochem. Physiol. 106C: 615-628.

Litaker, R.W., M. Vandersea, M. Faust, S. Kibler, M. Chinain, M.J. Holmes, W. Holland \& P. Tester. 2009. Taxonomy of Gambierdiscus including four new species, Gambierdiscus caribaeus, Gambierdiscus carolinianus, Gambierdiscus carpenter and Gambierdiscus ruetzleri (Gonyaulacales, Dinophyceae). Phycologia 8: 344-390.

Litaker, R.W., M. Vandersea, M. Faust, S. Kibler, A.W. Nau, W. Holland, M. Chinain, M.J. Holmes \& P.
Tester. 2010. Global distribution of ciguatera causing dinoflagellates in the genus Gambierdiscus. Toxicon 56: 711-730

Lizano, O.G. 2008. Algunas características dinámicas de las aguas alrededor de la Isla del Coco, Costa Rica. Rev. Biol. Trop. 56 (Supl. 2): 31-48.

Morales-Ramírez, A. \& E. Brugnoli-Olivera. 2001. El Niño 1997-1998 impact on the plankton dynamics in the Gulf of Nicoya, Pacific coast of Costa Rica. Rev. Biol. Trop. (Supl. 2): 103-114.

Pennington, J.T., K.L. Mahoney, V.S. Kuwahara, D.D. Kolber, R. Callienes \& F.P. Chavez. 2006. Primary production in the eastern tropical Pacific: a review. Prog. Oceanogr. 69: 285-317.

Rhodes, L. 2011. World-wide occurrence of the toxic dinoflagellate genus Ostreopsis Schmidt. Toxicon 57: 400-407.

Rojas, W. \& G.E. Alvarado. 2012. Geología y contexto geotectónico de la Isla del Coco y la zona marítima frente al Pacífico central de Costa Rica. Rev. Biol. Trop. 60 (Supl. 3): 15-32.

Sar, E.A., M. Ferrario \& B. Reguera. 2002. Floraciones algales nocivas en el Cono Sur Americano. Instituto Español de Oceanografía, Madrid, España.

Steidinger, K.A. 1993. Some taxonomic and biologic aspects of toxic dinoflagellates, pp. 1-28. In I.R. Falconer (Ed.). Algal Toxins in Seafood and Drinking Water. Academic Press, Londres.

Tester, P.A., R. Feldman, A.W. Nau, S.R. Kibler \& R.W. Litaker. 2010. Ciguatera fish poisoning and sea surface temperatures in the Caribbean Sea and the West Indies. Toxicon 56: 698-710.

Tindall, D.R. \& S.L. Morton. 1998. Community dynamics and physiology of epiphytic/benthic dinoflagellates associated with ciguatera, pp. 219-313. In D.M. Anderson, A.D. Cembella \& G.M. Hallegraeff (Eds.). Physiological Ecology of Harmful Algal Blooms. Springer, California.

Yasumoto, T., I. Nakajima, Y. Oshima \& R. Bagnis. 1979. A new toxic dinoflagellates found in association with ciguatera, pp. 65-70. In D.M. Anderson, A.W. White \& D.G. Baden (Eds.). Toxic Dinoflagellates. Elsevier, Nueva York.

Yasumoto, T., N. Seino, M. Yasutaka \& M. Murata. 1987. Toxins produced by benthic dinoflagellates. Biol. Bull. 173: 128-131. 
